\title{
Conditions for a Class of Entanglement Transformations
}

\author{
M. A. Nielsen* \\ Department of Physics, MC 12-33, California Institute of Technology, Pasadena, California 91125
}

(Received 2 December 1998)

\begin{abstract}
Suppose Alice and Bob jointly possess a pure state, $|\psi\rangle$. Using local operations on their respective systems and classical communication it may be possible for Alice and Bob to transform $|\psi\rangle$ into another joint state $|\phi\rangle$. This Letter gives necessary and sufficient conditions for this process of entanglement transformation to be possible. These conditions reveal a partial ordering on the entangled states and connect quantum entanglement to the algebraic theory of majorization. As a consequence, we find that there exist essentially different types of entanglement for bipartite quantum systems.
\end{abstract}

PACS numbers: 03.67.-a, 03.65.Bz

The question "What tasks may be accomplished using a given physical resource?" is of fundamental importance in many areas of physics. In particular, the burgeoning field of quantum information [1,2] is much concerned with understanding transformations between different types of quantum information. A fundamental example is the problem of entanglement transformation: Suppose $|\psi\rangle$ is a pure state of some composite system $A B$; we refer to system $A$ as Alice's system and to system $B$ as Bob's system. Into what class of states $|\phi\rangle$ may $|\psi\rangle$ be transformed, assuming that Alice and Bob may use only local operations on their respective systems, and unlimited two-way classical communication?

This Letter presents necessary and sufficient conditions for entanglement transformation to be possible. These conditions exhibit an unexpected connection between entanglement and the linear-algebraic theory of majorization. Furthermore, the existence of essentially different types of entanglement follows immediately from the conditions, together with a classification for the different types.

There has been extensive work on entanglement transformation. The problem was introduced in two guises by Bennett et al. [3-5]. They studied entanglement distillation, solving the problem of transforming some given pure state into (approximate) EPR pairs in the asymptotic limit where many identical copies of the pure state are initially available. They also studied the inverse procedure of entanglement formation, solving the problem of transforming EPR pairs into many (approximate) copies of some given pure state, again in the asymptotic limit. We will rederive these results as a consequence of the present investigation. In [3] the problem was also generalized to asymptotic and approximate transformation between mixed states and EPR pairs, and further results about these transformations were obtained in [4,5].

The investigations here are for the finite (nonasymptotic) case, from which asymptotic results may be recovered by taking limits. We do not directly consider approximate transformations.

Majorization is a large and active area of research in linear algebra, with entire books [6,7] devoted to its theory and application. We use Chap. 2 of Bhatia [10] as our principal reference on majorization. Suppose $x \equiv$ $\left(x_{1}, \ldots, x_{d}\right)$ and $y=\left(y_{1}, \ldots, y_{d}\right)$ are real $d$-dimensional vectors. Then $x$ is majorized by $y$ (equivalently $y$ majorizes $x$ ), written $x \prec y$, if for each $k$ in the range $1, \ldots, d$,

$$
\sum_{j=1}^{k} x_{j}^{\downarrow} \leq \sum_{j=1}^{k} y_{j}^{\downarrow},
$$

with equality holding when $k=d$, and where the $\downarrow$ indicates that elements are to be taken in descending order, so, for example, $x_{1}^{\downarrow}$ is the largest element in $\left(x_{1}, \ldots, x_{d}\right)$. The majorization relation is a partial order on real vectors, with $x \prec y$ and $y \prec x$ if and only if $x^{\downarrow}=y^{\downarrow}$.

To state our central result linking entanglement with majorization we need some notation. Suppose $|\psi\rangle$ is any state of Alice and Bob's system. $\rho_{\psi}$ denotes the state of Alice's system, that is, $\rho_{\psi} \equiv \operatorname{tr}_{B}(|\psi\rangle\langle\psi|)$. $\quad \lambda_{\psi}$ denotes the vector of eigenvalues of $\rho_{\psi} \cdot|\psi\rangle \rightarrow|\phi\rangle$, read " $|\psi\rangle$ transforms to $|\phi\rangle$ " indicates that $|\psi\rangle$ may be transformed into $|\phi\rangle$ by local operations and potentially unlimited two-way classical communication. Then we have the following:

Theorem 1: $|\psi\rangle$ transforms to $|\phi\rangle$ using local operations and classical communication if and only if $\lambda_{\psi}$ is majorized by $\lambda_{\phi}$. More succinctly,

$$
|\psi\rangle \rightarrow|\phi\rangle \text { iff } \lambda_{\psi} \prec \lambda_{\phi} .
$$

As a simple application of the result, suppose Alice and Bob each possess a three-dimensional quantum system, with respective orthonormal bases denoted $|1\rangle,|2\rangle,|3\rangle$. Define states $|\psi\rangle$ and $|\phi\rangle$ of their joint system by

$$
\begin{aligned}
& |\psi\rangle \equiv \sqrt{\frac{1}{2}}|11\rangle+\sqrt{\frac{2}{5}}|22\rangle+\sqrt{\frac{1}{10}}|33\rangle, \\
& |\phi\rangle \equiv \sqrt{\frac{3}{5}}|11\rangle+\sqrt{\frac{1}{5}}|22\rangle+\sqrt{\frac{1}{5}}|33\rangle .
\end{aligned}
$$

It follows from Theorem 1 that neither $|\psi\rangle \rightarrow|\phi\rangle$ nor $|\phi\rangle \rightarrow|\psi\rangle$, providing an example of essentially different types of entanglement, from the point of view of local 
operations and classical communication. We will say that $|\psi\rangle$ and $|\phi\rangle$ are incomparable. Bennett, Popescu, Rohrlich, and Smolin [8] have found specific examples of three-party entangled states which are incomparable in a similar sense.

To prove the theorem we first collect the following useful facts:

(i) Lo and Popescu [9] have shown that an arbitrary protocol transforming $|\psi\rangle$ to $|\phi\rangle$ using local operations and two-way classical communication may be simulated by a one-way communication protocol of the following form: Alice performs a generalized measurement on her system and then sends the result of her measurement to Bob, who performs an operation on his system, conditional on the measurement result.

(ii) For any matrix $A$, the polar decomposition [10] states that $A=\sqrt{A A^{\dagger}} U$, for some unitary $U$.

(iii) Suppose $\rho^{\prime}=\sum_{i} p_{i} U_{i} \rho U_{i}^{\dagger}$, where $p_{i} \geq 0$, $\sum_{i} p_{i}=1$, and the $U_{i}$ are unitary. Then the vector of eigenvalues of $\rho^{\prime}$ is majorized by the vector of eigenvalues of $\rho, \lambda_{\rho^{\prime}} \prec \lambda_{\rho}$, in an obvious notation [11].

(iv) Suppose $x \prec y$. Then $x=D y$, where $D$ is a matrix that may be written as a product of at most $d-1$ $T$ transforms, where $d$ is the dimension of $x$ and $y$ [10]. A $T$ transform, by definition, acts as the identity on all but two matrix components. On those two components it has the form

$$
T=\left[\begin{array}{cc}
t & 1-t \\
1-t & t
\end{array}\right],
$$

where $0 \leq t \leq 1$

(v) We make repeated use of the Schmidt decomposition [12]: Any pure state $|x\rangle$ of a composite system $A B$ may be written in the form $|x\rangle=\sum_{i} \sqrt{\lambda_{i}}\left|i_{A}\right\rangle\left|i_{B}\right\rangle$, where $0 \leq \lambda_{i}, \sum_{i} \lambda_{i}=1$, and $\left|i_{A}\right\rangle\left(\left|i_{B}\right\rangle\right)$ form an orthonormal basis for system $A(B)$. Note that $\rho_{x}$ has eigenvalues $\lambda_{i}$. Furthermore, we write $|x\rangle \sim|y\rangle$ if $|x\rangle$ and $|y\rangle$ are the same up to local unitary operations by Alice and Bob. The Schmidt decomposition implies that $|x\rangle \sim|y\rangle$ if and only if $\rho_{x}$ and $\rho_{y}$ have the same spectrum of eigenvalues.

Proof of Theorem 1: Suppose first that $|\psi\rangle \rightarrow|\phi\rangle$. Using fact (i) we assume that Alice performs a generalized measurement [13], described by operators $M_{m}$ on her system, satisfying the completeness relation $\sum_{m} M_{m}^{\dagger} M_{m}=I$, and then sends the result to Bob, who performs an operation $\mathcal{E}_{m}^{B}$, possibly nonunitary, on his system, conditional on the result $m$. Thus

$$
|\phi\rangle\langle\phi|=\sum_{m} \mathcal{E}_{m}^{B}\left(M_{m}|\psi\rangle\langle\psi| M_{m}^{\dagger}\right) .
$$

Since $|\phi\rangle$ is a pure state, it follows that

$$
\mathcal{E}_{m}^{B}\left(M_{m}|\psi\rangle\langle\psi| M_{m}^{\dagger}\right) \propto|\phi\rangle\langle\phi| .
$$

Tracing out system $B$ gives $M_{m} \rho_{\psi} M_{m}^{\dagger} \propto \rho_{\phi}$, with non-negative constants of proportionality $p_{m}$ satisfying $\sum_{m} p_{m}=1$. Polar decomposing $M_{m} \sqrt{\rho_{\psi}}$ gives

$$
M_{m} \sqrt{\rho_{\psi}}=\sqrt{M_{m} \rho_{\psi} M_{m}^{\dagger}} U_{m}=\sqrt{p_{m}} \sqrt{\rho_{\phi}} U_{m},
$$

where $U_{m}$ is a unitary matrix. But $\sum_{m} M_{m}^{\dagger} M_{m}=I$, from which we obtain

$$
\rho_{\psi}=\sum_{m} \sqrt{\rho_{\psi}} M_{m}^{\dagger} M_{m} \sqrt{\rho_{\psi}} .
$$

Substituting Eq. (8) and its adjoint into Eq. (9) gives $\rho_{\psi}=\sum_{m} p_{m} U_{m}^{\dagger} \rho_{\phi} U_{m}$, and fact (iii) implies that $\lambda_{\psi} \prec$ $\lambda_{\phi}$, as required.

To prove the converse, we consider first the twodimensional case, which demonstrates the essential idea of the general proof. Using orthonormal basis states $|0\rangle$ and $|1\rangle$, and the Schmidt decomposition, we may always write

$$
|\psi\rangle \sim\left|\psi^{\prime}\right\rangle=\sqrt{\alpha_{+}}|00\rangle+\sqrt{\alpha_{-}}|11\rangle,
$$

where $0 \leq \alpha_{-} \leq \alpha_{+} \leq 1$, and $\alpha_{+}+\alpha_{-}=1$. Since $\lambda_{\psi} \prec \lambda_{\phi}$, we may choose non-negative $\beta_{ \pm}$which sum to one, such that $\beta_{-} \leq \alpha_{-}$and $\alpha_{+} \leq \beta_{+}$, and

$$
|\phi\rangle \sim\left|\phi^{\prime}\right\rangle=\sqrt{\beta_{+}}|00\rangle+\sqrt{\beta_{-}}|11\rangle .
$$

The first step of the protocol is to transform $|\psi\rangle$ to $\left|\psi^{\prime}\right\rangle$, which Alice and Bob may do with local unitary operations. A simple eigenvalue calculation [fact (v)] shows that

$$
\left|\psi^{\prime}\right\rangle \sim\left|\psi^{\prime \prime}\right\rangle \equiv \frac{|00\rangle+|1\rangle[\cos (\gamma)|0\rangle+\sin (\gamma)|1\rangle]}{\sqrt{2}},
$$

where $\gamma$ is chosen to satisfy $\alpha_{+}=[1+\cos (\gamma)] / 2$. The next step of the protocol is for Alice and Bob to transform $\left|\psi^{\prime}\right\rangle$ to $\left|\psi^{\prime \prime}\right\rangle$, again by local unitary operations on their respective systems. Next, define operators $M_{1}$ and $M_{2}$ on Alice's system to have the following matrix representations in the $|0\rangle,|1\rangle$ basis:

$$
\begin{aligned}
& M_{1}=\left[\begin{array}{cc}
\cos (\delta) & 0 \\
0 & \sin (\delta)
\end{array}\right] ; \\
& M_{2}=\left[\begin{array}{cc}
\sin (\delta) & 0 \\
0 & \cos (\delta)
\end{array}\right] .
\end{aligned}
$$

$\delta$ is a parameter whose exact value will be fixed later in the proof. Note that $M_{1}^{\dagger} M_{1}+M_{2}^{\dagger} M_{2}=I$, so this defines a generalized measurement on Alice's system, which may be implemented using standard techniques involving only projective measurements and unitary transforms [14]. Let $\left|\psi_{m}^{\prime \prime \prime}\right\rangle$ denote the state after the measurement, given that outcome $m$ occurred. Then

$$
\begin{aligned}
\left|\psi_{1}^{\prime \prime \prime}\right\rangle= & \cos (\delta)|00\rangle+\sin (\delta)|1\rangle \\
& \times[\cos (\gamma)|0\rangle+\sin (\gamma)|1\rangle], \\
\left|\psi_{2}^{\prime \prime \prime}\right\rangle= & \sin (\delta)|00\rangle+\cos (\delta)|1\rangle \\
& \times[\cos (\gamma)|0\rangle+\sin (\gamma)|1\rangle] .
\end{aligned}
$$


By symmetry or explicit eigenvalue calculation, one may verify that $\left|\psi_{1}^{\prime \prime \prime}\right\rangle \sim\left|\psi_{2}^{\prime \prime \prime}\right\rangle$. Thus Alice and Bob can ensure that the final state is $\left|\psi^{\prime \prime \prime}\right\rangle \equiv\left|\psi_{1}^{\prime \prime \prime}\right\rangle$, by applying appropriate unitary transforms to their respective systems. To do this, Alice must send her measurement result to Bob, so he knows which unitary operation to apply. An eigenvalue calculation shows that

$$
\left|\psi^{\prime \prime \prime}\right\rangle \sim \sqrt{\lambda_{+}}|00\rangle+\sqrt{\lambda_{-}}|11\rangle,
$$

where

$$
\lambda_{ \pm} \equiv \frac{1 \pm \sqrt{1-\sin ^{2}(2 \delta) \sin ^{2}(\gamma)}}{2} .
$$

At $\delta=0, \quad \lambda_{+}=1 \quad$ and $\quad$ at $\delta=\pi / 4, \quad \lambda_{+}=$ $[1+\cos (\gamma)] / 2=\alpha_{+}$. Since $\alpha_{+} \leq \beta_{+} \leq 1$, continuity ensures that the equation $\lambda_{+}(\delta)=\beta_{+}$has a real solution $\delta=\frac{1}{2} \arcsin \left[2\left(\beta_{+}-\beta_{+}^{2}\right)^{1 / 2} / \sin \gamma\right]$. Choosing this $\delta$ gives $\left|\psi^{\prime \prime \prime}\right\rangle \sim\left|\phi^{\prime}\right\rangle=\sqrt{\beta_{+}}|00\rangle+\sqrt{\beta_{-}}|11\rangle$, and therefore by applying local unitary transformations Alice and Bob may obtain the state $\left|\phi^{\prime}\right\rangle$, and from there the state $|\phi\rangle$, by Eq. (11).

The general case uses fact (iv) to reduce the problem to the two-dimensional case by cascading a sequence of entanglement transformations, each corresponding to a single $T$ transform. Using facts (iv) and (v) we may assume that Alice and Bob are each in possession of a $d$-dimensional system, with orthonormal bases $|0\rangle,|1\rangle, \ldots,|d-1\rangle$, that the state $|\psi\rangle$ has the form

$$
\begin{aligned}
|\psi\rangle \sim\left|\psi^{\prime}\right\rangle= & \cos (\zeta)\left(\sqrt{\alpha_{+}}|00\rangle+\sqrt{\alpha_{-}}|11\rangle\right) \\
& +\sin (\zeta)\left|\psi_{\perp}\right\rangle
\end{aligned}
$$

where $\left|\psi_{\perp}\right\rangle$ is a normalized state of the form $\sum_{j=2}^{d-1} \psi_{j}|j\rangle|j\rangle, \zeta$ is real, and

$$
\begin{aligned}
|\phi\rangle \sim\left|\phi^{\prime}\right\rangle= & \cos (\zeta)\left(\sqrt{\beta_{+}}|00\rangle+\sqrt{\beta_{-}}|11\rangle\right) \\
& +\sin (\zeta)\left|\psi_{\perp}\right\rangle .
\end{aligned}
$$

$0 \leq \beta_{-} \leq \alpha_{-} \leq \alpha_{+} \leq \beta_{+}$, as before. The $T$ transform corresponds to a transformation of the $|00\rangle$ and $|11\rangle$ terms in these expressions. The protocol is as for the twodimensional case, except for a slight change at the measurement stage. Alice does a generalized measurement described by operators $\tilde{M}_{1}$ and $\tilde{M}_{2}$ defined in terms of the earlier operators $M_{1}$ and $M_{2}$ by

$$
\tilde{M}_{1}=\left[\begin{array}{cc}
M_{1} & 0 \\
0 & \frac{I_{d-2}}{\sqrt{2}}
\end{array}\right] ; \quad \tilde{M}_{2}=\left[\begin{array}{cc}
M_{2} & 0 \\
0 & \frac{I_{d-2}}{\sqrt{2}}
\end{array}\right] .
$$

The matrices $I_{d-2} / \sqrt{2}$ in the lower right hand corner ensure that coherence is preserved during the transformation procedure, and the completeness relation $\sum_{m} \tilde{M}_{m}^{\dagger} \tilde{M}_{m}=I$ is obeyed. With this change the protocol proceeds as before to transform $|\psi\rangle$ to $|\phi\rangle$.

The next few paragraphs examine some consequences of Theorem 1. Note first that the proof of the theorem, together with the method given in [10] for obtaining $\lambda_{\psi}$ from $T$ transforms acting on $\lambda_{\phi}$, gives a constructive method involving at most $d-1$ bits of communication to transform $|\psi\rangle$ to $|\phi\rangle$, whenever $\lambda_{\psi} \prec \lambda_{\phi}$.
Generalizing the earlier example of incomparable states $|\psi\rangle$ and $|\phi\rangle$, I conjecture that in the limit where $A$ and $B$ are of large dimensionality, almost all pairs of pure states $|\psi\rangle$ and $|\phi\rangle$ picked according to the unitary invariant measure on $A B$ [15] will be incomparable. A heuristic argument is as follows. Let $p_{i}$ and $q_{i}$ be random variables denoting the eigenvalues of $\rho_{\psi}$ and $\rho_{\phi}$, arranged into decreasing order. Define $\Delta_{i} \equiv p_{i}-q_{i}$. Then $|\psi\rangle$ and $|\phi\rangle$ are incomparable if (and only if) the stochastic process $T_{k} \equiv \sum_{i=1}^{k} \Delta_{i}$ crosses the origin; that is, it is positive for some values of $k$, and negative for others. If $T_{k}$ were a random walk with independent and identically distributed increments, the conjecture would be true in the limit of large dimension [16]. $T_{k}$ fails to be a random walk for two reasons: (a) The ordering of the $p_{i}$ and $q_{i}$ ensures that the typical size of the increments $\Delta_{i}$ tends to decrease as $i$ gets larger and (b) the constraint $\sum_{i=1}^{d} \Delta_{i}=0$ ensures that the increments are correlated. Intuitively, in the limit of large dimensionality, the distribution of the $\Delta_{i}$ becomes very nearly uncorrelated from step to step, with the remaining correlations acting as a weak "restoring force" towards the origin, which tends to enhance crossings. Furthermore, the distribution "flattens out" in large dimensions, with only a very slow decrease in the typical size of the increments [17]. So in large dimensions the $\Delta_{i}$ behave locally like increments of a random walk, which can therefore be expected to cross the origin.

Theorem 1 allows the well-developed theory of isotone functions [10] to be applied to the study of entanglement. For example, an important subclass of the isotone functions is the Schur-convex functions: $f: R^{d} \rightarrow R$ such that $x \prec y$ implies $f(x) \leq f(y)$. Well-known Schur-convex functions [10] include the maps $\left\{x_{i}\right\} \rightarrow \sum_{i} x_{i} \log x_{i}$ and $\left\{x_{i}\right\} \rightarrow \sum_{i} x_{i}^{k}$, for any $k \geq 1$. It follows that if $|\psi\rangle \rightarrow$ $|\phi\rangle$, then $S\left(\rho_{\phi}\right) \leq S\left(\rho_{\psi}\right)$, where $S(\cdot)$ is the von Neumann entropy, and $\operatorname{tr}\left(\rho_{\psi}^{k}\right) \leq \operatorname{tr}\left(\rho_{\phi}^{k}\right)$, for any $k \geq 1$.

Theorem 1 simplifies in the special case where Alice's system is two dimensional, and Bob's system arbitrary, telling us that $|\psi\rangle \rightarrow|\phi\rangle$ if and only if $S\left(\rho_{\phi}\right) \leq S\left(\rho_{\psi}\right)$.

Theorem 1 may be combined with the asymptotic equipartition theorem (Chap. 3 of [18]) to provide a straightforward proof of some results of Bennett et al. [3]. They showed how to approximately transform back and forth between $n$ copies of the state $|\phi\rangle$ and $n S\left(\rho_{\phi}\right)$ EPR pairs, in the limit where $n$ becomes large. The following is a sketch of the proof based upon Theorem 1 .

Suppose Alice and Bob share $m$ EPR pairs. Denote their total state by $|\psi\rangle$, which has a corresponding vector of eigenvalues $\left(2^{-m}, 2^{-m}, \ldots, 2^{-m}\right)$. Let $|\phi\rangle$ be any pure state of $A B$. Taking $n$ copies of $|\phi\rangle$, the asymptotic equipartition theorem implies that for sufficiently large $n$ the state may be approximated by just $2^{n S\left(\rho_{\phi}\right)}$ terms in the Schmidt decomposition,

$$
|\phi\rangle^{\otimes n} \approx\left|\phi^{\prime}\right\rangle \equiv \sum_{i=1}^{2^{n S\left(\rho_{\phi}\right)}} \sqrt{\lambda_{i}}|i\rangle|i\rangle .
$$


Choose $m$ such that $m \approx n S\left(\rho_{\phi}\right)$. Then it is easy to check directly that $\left(2^{-m}, \ldots, 2^{-m}\right) \prec\left(\lambda_{1}, \ldots, \lambda_{2^{n S\left(\rho_{\phi}\right)}}\right)$, so Theorem 1 implies that $|\psi\rangle \rightarrow\left|\phi^{\prime}\right\rangle$, and thus it is possible to transform $n S\left(\rho_{\phi}\right)$ EPR pairs into a pretty good approximation to $n$ copies of $|\phi\rangle$.

For the inverse procedure note that by the asymptotic equipartition theorem there is a set of roughly $2^{n S\left(\rho_{\phi}\right)}$ terms in the Schmidt decomposition such that

$$
|\phi\rangle^{\otimes n} \approx \sum^{\prime} \sqrt{\lambda_{i}}|i\rangle|i\rangle,
$$

where the primed sum indicates that we are summing over a restricted set where $\lambda_{i} \lesssim 2^{-n S\left(\rho_{\phi}\right)}$. The transformation procedure is for Alice to first project onto the space spanned by the terms $|i\rangle$ appearing in the sum. This succeeds with probability $1-\epsilon \approx 1$, leaving the state in the form

$$
\sum^{\prime} \sqrt{\frac{\lambda_{i}}{1-\epsilon}}|i\rangle|i\rangle
$$

Therefore, for any $m$ such that $2^{-n S\left(\rho_{\phi}\right)} /(1-\epsilon) \leq 2^{-m}$ Theorem 1 implies that the $n$ copies of $|\phi\rangle$ may be transformed to $m$ EPR pairs. In particular, we may choose $m \approx n S\left(\rho_{\phi}\right)$.

There are many open problems to which Theorem 1 may be of relevance. It would be of great interest to determine when a mixed state $\rho$ can be transformed to a mixed state $\sigma$ by local operations and classical communication. This would also provide a good starting point to better understand approximate entanglement transformation. A related problem is to determine transformation conditions for three- (or more) party pure state entanglement analogous to those found here for two party entanglement. Finally, I hope that the connection between entanglement and majorization may enable us to better understand the fundamental measures of entanglement introduced by Wootters and collaborators $[5,19]$.

I thank Howard Barnum, who introduced me to the beautiful subject of majorization, Dorit Aharonov and Bill Wootters, whose words and papers convinced me that entanglement for its own sake is a deeply interesting subject, and Ike Chuang, Chris Fuchs, Julia Kempe, and John Preskill for helpful discussions. This work was supported by DARPA through the Quantum Information and Computing Institute (QUIC) administered through the ARO.

*Email address: mnielsen@theory.caltech.edu

[1] C. H. Bennett, Phys. Today 48, No. 10, 24 (1995).

[2] J. Preskill, Proc. R. Soc. London A 454, 469 (1998).

[3] C. H. Bennett, H. J. Bernstein, S. Popescu, and B. Schumacher, Phys. Rev. A 53, 2046 (1996).

[4] C. H. Bennett et al., Phys. Rev. Lett. 76, 722 (1996).

[5] C. H. Bennett, D. P. DiVincenzo, J. A. Smolin, and W. K. Wootters, Phys. Rev. A 54, 3824 (1996).

[6] A. W. Marshall and I. Olkin, Inequalities: Theory of Majorization and Its Applications (Academic Press, New York, 1979).

[7] P. M. Alberti and A. Uhlmann, Stochasticity and Partial Order: Doubly Stochastic Maps and Unitary Mixing (Dordrecht, Boston, 1982).

[8] C. H. Bennett, S. Popescu, D. Rohrlich, and J. Smolin, report.

[9] H.-K. Lo and S. Popescu, quant-ph/9707038.

[10] R. Bhatia, Matrix Analysis (Springer-Verlag, New York, 1997).

[11] A. Uhlmann, Wiss. Z. Karl-Marx-Univ. Leipzig 20, 633 (1971).

[12] A. Peres, Quantum Theory: Concepts and Methods (Kluwer Academic, Dordrecht, 1993).

[13] C.W. Gardiner, Quantum Noise (Springer-Verlag, Berlin, 1991).

[14] B. W. Schumacher, Phys. Rev. A 54, 2614 (1996).

[15] D. N. Page, Phys. Rev. Lett. 71, 1291 (1993).

[16] G. R. Grimmett and D. R. Stirzaker, Probability and Random Processes (Clarendon Press, Oxford, 1992).

[17] E. Lubkin, J. Math. Phys. (N.Y.) 19, 1028 (1978).

[18] T. M. Cover and J. A. Thomas, Elements of Information Theory (John Wiley and Sons, New York, 1991).

[19] W. K. Wootters, Phys. Rev. Lett. 80, 2245 (1998). 